\title{
On the mechanism of the shape elongation of embedded nanoparticles
}

\section{Amekura, $\mathrm{H}$.}

2020-07-15

Amekura , H , Kluth , P , Mota-Santiago , P , Jantunen , V , Leino , A A, Vazquez , H , Nordlund, K, Djurabekova , F \& Sahlberg , I 2020 , ' On the mechanism of the shape elongation of embedded nanoparticles ', Nuclear Instruments \& Methods in Physics

Research. Section B: Beam Interactions with Materials and Atoms , vol. 475 , pp. 44-48 . https://doi.org/10.1016/j.nin

http://hdl.handle.net/10138/325126

https://doi.org/10.1016/j.nimb.2020.04.038

cc_by_nc_nd

submittedVersion

Downloaded from Helda, University of Helsinki institutional repository.

This is an electronic reprint of the original article.

This reprint may differ from the original in pagination and typographic detail.

Please cite the original version. 


\section{On the mechanism of the shape elongation of embedded nanoparticles}

H. Amekura ${ }^{1)^{*}}$, P. Kluth ${ }^{2)}$, P. Mota-Santiago ${ }^{2)}$, I. Sahlberg ${ }^{3)}$, V. Jantunen ${ }^{3)}$, A. A. Leino ${ }^{3)}$, H. Vazquez ${ }^{3)}$, K. Nordlund ${ }^{3)}$, and F. Djurabekova ${ }^{3)}$

1) National Institute for Materials Science (NIMS), Tsukuba, Ibaraki, Japan

2) Department of Electronic Materials Engineering, Research School of Physics, Australian National University (ANU), Canberra, Australia

${ }^{3)}$ Helsinki Institute of Physics and Department of Physics, University of Helsinki, Helsinki, Finland

Abstract: The mechanism of the shape elongation of metal nanoparticles (NPs) in amorphous silica, which is induced under swift heavy ion irradiation, is discussed. Since the discovery of this phenomenon, several mechanisms were proposed and debated. Now, only two major mechanisms have survived: (i) the synergy model between the ion hammering and the transient melting of NPs by the inelastic thermal spike, and (ii) the thermal pressure and flow model. Here, we discuss that three experimental results are inconsistent with (i). The latter is supported by two-temperature molecular dynamics simulations (TT-MD), which simulate not only the atomic motions but also the local electron temperatures. While a remarkable correlation was observed between the temporal evolution of the silica density around the ion trajectory and that of the aspect ratio of the NP later than $\sim 1$ ps after the ion impact, no correlation was observed earlier than $\sim 1$ ps. Since the silica has a much higher electronlattice (e-L) coupling than the metal NP, the lattice temperature quickly increases up to remarkably high values, which results in quick and large expansion and recovery in silica. By contrast, metal NPs have low e-L coupling, which results in slow temperature change. The NP remains in a solid state in the period where silica experiences the quick expansion, and only melts and deforms when the silica is already in the recovery stage. The large difference of the temperature evolution between silica and metal NPs is the origin of the shape elongation.

Keywords: shape elongation, ion shaping, nanoparticle, swift heavy ion, two-temperature molecular dynamics 


\section{REI 2019}

Nur-Sultan (Kazakhstan) August 19-23, 2019

\section{Introduction}

The shape elongation of nanoparticles (NPs) was firstly observed by D'Orleans et al. in 2003 under swift heavy ion (SHI) irradiation of $200 \mathrm{MeV}$ I [1]. They formed Co NPs with a mean diameter of $\sim 10$ nm by implantation with $160 \mathrm{keV}$ Co ions into $300 \mathrm{~nm}$ thick $\mathrm{SiO}_{2}$ layers on $\mathrm{Si}$ at elevated temperature of $873 \mathrm{~K}$. Large NPs were required for observing the shape elongation, because it was proposed that the elongation was induced only for NPs larger than the ion track diameter [2]. The large size was considered a prerequisite for the shape elongation of NPs. In the original paper [1], NPs were irradiated with $200 \mathrm{MeV}{ }^{127} \mathrm{I}$ ions to fluences up to $1 \times 10^{14}$ ions $/ \mathrm{cm}^{2}$ at room temperature, which resulted in a drastic change of the shape of NPs. They were found to be strongly elongated along the same direction as the SHI beam.

Soon after the discovery, the same phenomenon was observed in a different configuration, i.e. in chemically-synthesized free-standing Au-core/silica-shell colloidal NPs [3]. After irradiation with 30 $\mathrm{MeV}$ selenium ions to a fluence of $2 \times 10^{14}$ ions $/ \mathrm{cm}^{2}$, the spherical gold core of $14 \mathrm{~nm}$ in diameter elongated along the beam direction and transformed to a rod of $6 \mathrm{~nm}$ diameter and $54 \mathrm{~nm}$ length. Simultaneously the silica shell expanded perpendicularly to the beam and shrank in the direction parallel to the beam. Since colloidal silica NPs without $\mathrm{Au}$ cores show the similar shape changes, the deformation of the silica shell was ascribed to the ion hammering [4]. To elucidate the deformation mechanism, a series of core/shell NPs was prepared with the thickness of silica shell ranging from 15 and $72 \mathrm{~nm}$, while the diameter of the Au core was kept the same $14 \mathrm{~nm}$. No measurable deformation of the Au core was confirmed for the shells thinner than $26 \mathrm{~nm}$ under $30 \mathrm{MeV}$ Se irradiation. It was concluded that silica shells play a major role in the deformation of the Au cores. The driving force for deformation of the Au cores was believed to be the ion hammering effect of the silica shells.

However, ion hammering can build up stress up the order of $100 \mathrm{MPa}$ only, which is too low to induce clear deformation of solid Au NPs. Klaumünzer pointed out in Ref. [5] that the observed large elongation of NPs cannot be explained by any known mechanisms of radiation-induced softening. 


\section{REI 2019}

Consequently a proposal was made of a synergy effect between the stress generated by the ion hammering and the transient melting of NPs by the inelastic thermal spike (i-TS) [6]. In this paper, we critically re-visit this synergy model.

Independently, Leino et al. [7] succeeded to simulate the process of elongation of an embedded Au NP numerically by employing the classical molecular dynamics (MD) simulations method combined with the i-TS model. In this way, the energy deposited in the structure by hot electrons through the electron-lattice interaction can affect the atomic dynamics directly within the MD cell. The authors described the mechanism as the "thermal pressure and flow" model, since they observed a flow of atoms of the NP under the pressure of strong thermal expansion. This model reproduced the elongation of NPs without assuming additional stress. Furthermore, while the additional stress was applied, which was expected from the hammering effect, the results did not drastically change, indicating that the hammering effect is not the dominant one. Using a similar methodology, we have numerically shown that the core-shell $(\mathrm{C} / \mathrm{S})$ ion tracks in silica are formed even without vaporization which is induced by the i-TS effect, supporting our experimental results [8]. In the course of the calculations, we have observed a temporal coincidence between the formation of the C/S track and the elongation of NPs [8]. Physical interpretation of the calculated results of the shape elongation of NPs and the C/S track formation are provided in this paper.

\section{Method}

Numerical simulations of NP elongation in silica were carried out using the classical MD [9] code PARCAS [10][11-14], previously widely used to study radiation effects, including swift heavy ions [11,15-17]. To initiate the ion track, we followed the practice of instantaneous energy deposition according to a profile obtained from the two-temperature iTS model [18]. This approach has been previously found to give good results for track radii [19] as well as NP elongation [16]. In this approach, all the atoms in the center of the material are given a certain amount of kinetic energy depending on 


\section{REI 2019}

107 their distance to the axis of the swift heavy ion passage. The energy deposition takes the form of some

108 suitable increase in the velocity of the particles. The direction and the magnitude of the velocity increase 109 is given at random.

110 In the current simulations, $\mathrm{Zn} \mathrm{NP}$ was embedded in an amorphous silica $\left(\mathrm{a}-\mathrm{SiO}_{2}\right)$ structure as 111 follows. A sphere of $12 \mathrm{~nm}$ in diameter, i.e., a NP, was cut out of the bulk Zn crystal structure relaxed 112 at zero pressure and room temperature. The NP was compressed by 2 per cent, following the procedure 113 described in [20]. The compressed NP was inserted into a cavity of the same size and shape in the center 114 of the $\mathrm{a}_{-} \mathrm{SiO}_{2}$ cell. The combined structure was relaxed again under pressure control at $300 \mathrm{~K}$, allowing 115 the whole structure to reach the equilibrium. During this time, the compressed NP expanded in the 116 cavity, simultaneously interacting with the a- $\mathrm{SiO}_{2}$ structure and establishing natural bonds with the 117 surrounding atoms. We used the Tersoff-like Munetoh potential for $\mathrm{SiO}_{2}$ [21] combined with the 118 Tersoff-like Zn potential from [22].

119 The time for all simulations was $100 \mathrm{ps}$; there was no need for longer simulation times, since the simulated systems did not change significantly during the latter half of the simulated period. We investigated the density distribution of the $\mathrm{SiO}_{2}$ above and below the NPs, in and around the formed ion track, in addition to the shape elongation of the NP. These density variations proved to be of key importance for the mechanism behind the observed elongation. The simulation cell was divided into cylindrical shells of width $1 \mathrm{~nm}$, and the radial distribution of the density was analyzed by comparing the values for the different shells.

\section{Results and Discussion}

\subsection{Criticism of the Synergy model from experimental results}

129 Here, we point out three inconsistencies of the synergy model with the experimental results:

130 a) The elongation angle vs. the beam incident angle

131 Recently, Slablab et al. irradiated $\mathrm{Au}$ NPs in $\mathrm{SiO}_{2}$ with different incident angles of $0^{\circ}, 30^{\circ}, 45^{\circ}$, and $13260^{\circ}$, and observed that the elongation angles are the same as the beam incident angles, by both 
133

transmission electron microscopy (TEM) and second-harmonic generation microscopy [23]. This

134 observation is inconsistent with the synergy model. The strain rate tensor $\mathrm{d} \varepsilon / \mathrm{d} t$ for the hammering is 135 given as

$$
\mathrm{d} \varepsilon / \mathrm{d} t=A \mathrm{~d} \Phi / \mathrm{d} t
$$

where $\Phi$ and $t$ denote the ion fluence and time, respectively. The angler dependence of the deformation tensor $A$ is given as [24],

$$
A=A_{o}\left(S_{e}, T_{i}\right)\left(\begin{array}{ccc}
1-3 \sin ^{2} \theta & 0 & 3 \sin \theta \cos \theta \\
0 & 1 & 0 \\
3 \sin \theta \cos \theta & 0 & 1-3 \cos ^{2} \theta
\end{array}\right)
$$

where the beam parallel to the $\mathrm{z}$ axis is tilted around the $\mathrm{y}$ axis by an angle $\theta$. Except $\theta=0^{\circ}$ and $90^{\circ}$, the off-diagonal parts do not vanish, i.e., something like rotations can be induced. Consequently, the beam incident angle and the elongation angle should be different, except $\theta=0^{\circ}$ and $90^{\circ}$. In fact, a self-standing silica colloid, which is governed by the ion hammering, changed the elongation angle from $45^{\circ}$ at $1 \times$ $10^{14}$ ions $/ \mathrm{cm}^{2}$ to $30^{\circ}$ at $8 \times 10^{14}$ ions $/ \mathrm{cm}^{2}$ under $4 \mathrm{MeV}$ Xe irradiation with the incident angle of $45^{\circ}[25,26]$. The observation of the same angle between the beam incidence and the elongation is counterevidence to the synergy model.

b) Non-existence of the threshold fluence for the elongation

Here we discuss the threshold fluence, below which the elongation is not induced. In fact, the threshold fluences have been reported from TEM observation [1,27] and RBS measurements [27]. The observed thresholds can be ascribed to the resolution limits of the detection methods. In most of cases, NPs are not completely spherical even before the SHI irradiation. To detect small elongation at low fluences, it is necessary to average the shapes over many NPs. Averaging over a macroscopic number of NPs is not inherently practical for TEM, which observes independent nanoclusters. In the case of RBS, the poor energy-resolution of the conventional surface barrier detector limits the elongation resolution. To overcome this problem, we have evaluated the anisotropy in the optical absorption of 


\section{REI 2019}

157 linearly polarized light, i.e., the optical linear dichroism (OLD). This method detects signals averaged 158 over a large number of NPs, and is consequently quite sensitive to the small elongation of NPs. While 159 the signal was null for the unirradiated state, a very weak anisotropy was detected at the fluence of $1 \times$ $16010^{11}$ ions $/ \mathrm{cm}^{2}$ under $200 \mathrm{MeV} \mathrm{Xe}{ }^{14+}$ ion irradiation [28]. Judging from the track radius of $\sim 4.5 \mathrm{~nm}$, this

161 fluence corresponds the track coverage of only 5\% of the surface area; i.e. most NPs are not impacted 162 at all, or impacted with the ion only once, not twice or more. This observation indicates that even only 163 one impact of the ion induces a small but certain elongation in NPs, i.e. the non-existence of the 164 threshold fluence. In the synergy model, the melting of NPs does not result in elongation until a 165 sufficient stress field is accumulated by the ion hammering. Therefore, a threshold fluence was expected 166 in the synergy model. However a threshold fluence was not experimentally observed, indicating the 167 exclusion of the synergy model [29].

168 A criticism on the OLD detection was whether the anisotropic signals really come from the NP 169 elongation or not. In fact, the observed OLD spectra well matched with the calculated spectra of Zn NPs 170 from Rayleigh theory [28], which supports the assignment of the observed anisotropy to the NP 171 elongation. However, the signal could be ascribed to optically anisotropic defects which are generated 172 by single impacts of the SHI ion. To exclude this possibility, we have carried out the same OLD 173 measurements for the same samples but without NPs, i.e., $\mathrm{SiO}_{2}$ only, irradiated with the same conditions.

174 We have observed that the OLD signal increases with the fluence even without NPs, but the intensity 175 was $\sim 2$ orders of the magnitude lower than the signal from the NPs, indicating that the observed OLD 176 signal is ascribed to the NPs [30].

$178 \quad$ c) Irradiation temperature dependence

179 It is known that ion hammering exhibits large irradiation temperature dependence, which 180 monotonically decreases with the irradiation temperature [31]. We are now evaluating the irradiation 181 temperature dependence of the shape elongation. While this is still preliminary, the elongation 


\section{REI 2019}

182 efficiency is almost constant between 300 and $600 \mathrm{~K}$ [32], whereas a steep decrease was reported [31]

183 for the ion hammering.

\subsection{The results of the MD simulations}

Figure 1 shows the time evolution of the $\mathrm{C} / \mathrm{S}$ track formation and the elongation of a $\mathrm{Zn} \mathrm{NP}$ in amorphous $\mathrm{SiO}_{2}$ irradiated with a $60 \mathrm{MeV} \mathrm{Ti}$ ion. Blue and green curves indicate relative density of $\mathrm{SiO}_{2}$ in the innermost cylinder of $1 \mathrm{~nm}$ radius along the ion trajectory (blue) and in the second shell of $1 \mathrm{~nm}$ thick (green). A red curve shows the aspect ratio of NP (see the right axis). Figure 2 schematically depicts the processes of the C/S track formation (upper row) and those of the shape elongation of NP (lower row), which were interpreted from the numerical results shown in Fig. 1 and physical insight.

At the moment of the ion impact $(t=0)$, the blue and the green curves were at unity of the relative density in the left axis, and the red curve is at unity of the aspect ratio in the right axis. Both the blue and green curves exhibits steep density drops, i.e., steep expansion, within less than 1 ps. These expansions are due to huge energy deposition from a SHI, which first excites the electronic system of silica and then transferred to the lattice system. Soon the expansion turns to density recovery with emitting a pressure wave outside. The emission of the pressure wave is also reported by Cherednikov et al. using the hybrid particle-in-cell/ MD simulations of a SHI impact in LiF [33]. While the curves shown in Fig. 1 are noisy, the noise could be partly ascribed to sound waves triggered by the pressure wave. To clarify this point, further study is necessary. These processes are schematically depicted in the upper row of Fig. 2. An interesting feature that can be observed in Fig. 1 is that the density in the innermost cylinder (green) is always lower than in the second shell (blue) except in the very beginning of the evolution. However, this observation does not always indicate the formation of the C/S track, because the shell density is lower than the unirradiated value, i.e., unity, at $t<20 \mathrm{ps}$. The track could be categorized into two temporal regions: The dynamical region below $40 \mathrm{ps}$, where the track is formed but the densities of both the 1 st and 2 nd shells change, and the static region exceeding $40 \mathrm{ps,} \mathrm{where}$ constant densities in the C/S track are observed. The lower density of the core compared to that of the 


\section{REI 2019}

shell in the dynamic region can be ascribed to the fact that the core is hotter than the shell. However, it could be difficult to explain the lower core density at $100 \mathrm{ps}$. The C/S structure was frozen, probably due to the extremely rapid cooling following the SHI impact.

Let us now consider the shape elongation of the NP. During the first $1 \mathrm{ps}$, the aspect ratio maintained almost at a constant value of nearly unity, while the surrounding silica experienced the steep expansion. In the MD simulations, the NP is instantaneously heated. However, due to the high mass of the $\mathrm{Zn}$ atoms, atom flow is not instantaneous, but needs some time to commence. Furthermore, the different temperature evolutions of silica and the NP may contribute to hinder the expansion of NP in the early stage. As shown in the 3-dimensional i-TS calculations reported by Rizza et al. [34], the lattice temperature $\left(T_{\mathrm{L}}\right)$ in the silica region along the ion trajectory is already high at $10-100 \mathrm{fs}$, while that of the NP is still very low. The electron temperatures $\left(T_{\mathrm{e}}\right)$ of both the silica and NP are much higher. This is because the electron-lattice (e-L) coupling is much higher in silica than in metal. Although $T_{\mathrm{e}}$ of NP is very high, the heat is not efficiently transferred to the lattice because of the low e- $\mathrm{L}$ coupling. Contrarily, $T_{\mathrm{e}}$ of silica is easily transferred to the lattice system, which results in a high $T_{\mathrm{L}}$. The heating of NP is rather induced at the boundary between the NP and silica, where hot electrons from the metal NP penetrate through the boundary and heat up the lattice of silica via the high e-L coupling of silica. Finally, the NP is heated up by thermal conduction from the hot silica region around the boundary, which were heated up by hot electrons from NP. Because of the outer boundary heating, the NP is fully heated up only after the initial energy deposition, and requires $\sim 3$ ps for complete melting. Since the strong expansion of silica completes within $1 \mathrm{ps}$, all the dynamics in the NP, such as the increase of temperature, melting and flow, happens during the density recovery stage of silica. Following the recovery flow and the $\mathrm{C} / \mathrm{S}$ track formation, an anisotropic deformation of NP, i.e., the shape elongation, is induced. The elongation processes are schematically depicted in the lower row of Fig. 2. Notable differences in the e-L couplings between silica and metal NPs are the origin of the shape elongation of NPs. 


\section{REI 2019}

Nur-Sultan (Kazakhstan) August 19-23, 2019

\section{Conclusions}

The mechanisms of the shape elongation of metal NPs embedded in amorphous silica, which is induced under SHI irradiation, were discussed in this paper. Since the discovery of this phenomenon in 2003, many publications have proposed different candidate mechanisms. Now only two major mechanisms survived : (i) the synergy model between the ion hammering and the transient melting of NPs by the inelastic thermal spike, and (ii) the thermal pressure and flow model. As pointed out, experimental results are inconsistent with the former: (a) the coincidence between the incident SHI beam angle and the elongation angle, and (b) non-existence of the threshold fluence for the elongation detected by the optical linear dichroism (OLD). This paper further points out that the irradiation-induced OLD signal from silica exists but was two orders of the magnitude lower than those of NPs. The nonexistence of the threshold fluence is further supported.

The model (ii) is supported by the molecular dynamic simulations with the lattice heating obtained from the inelastic thermal spike model. While a remarkable correlation was observed between the temporal evolution of the silica density around the ion trajectory and that of the aspect ratio of the NP after the first ps of the ion impact, no correlation was observed within $\sim 1$ ps. Since silica has much higher e-L coupling, the lattice temperature also increases quickly and up to higher values. Contrarily, metal NPs have the low e-L coupling, which results in slow temperature exchange. Hence the melting takes some time to complete, and the NP maintains itself in a still nearly solid state in the period where silica experiences the rapid expansion, and the NP melts and flows only in the course of the density recovery stage. Large difference of the temperature evolutions between silica and NPs is the origin of the shape elongation.

\section{Acknowledgments}

HA was supported by JSPS-KAKENHI Grant number 18K04898. IS, VJ, FD and KN gratefully acknowledge financial support from the Academy of Finland MESIOS and NANOIS projects, and CPU 
capacity grants from the IT Centre for Science CSC in Espoo, Finland. P.K. acknowledges the

260 Australian Research Council for financial support.

\section{Reference}

[1] C. D’Orleans, J.P. Stoquert, C. Estournès, C. Cerruti, J.J. Grob, J.L. Guille, F. Haas, D. Muller, and M. Richard-Plouet, Anisotropy of Co nanoparticles induced by swift heavy ions, Phys. Rev. B 67, 220101 (2003).

[2] M.C. Ridgway, R. Giulian, D.J. Sprouster, P. Kluth, L.L. Araujo, D.J. Llewellyn, A.P. Byrne, F. Kremer, P.F.P. Fichtner, G. Rizza, H. Amekura, and M. Toulemonde, Role of Thermodynamics in the Shape Transformation of Embedded Metal Nanoparticles Induced by Swift Heavy-Ion Irradiation, Phys. Rev. Lett. 106, 095505 (2011).

[3] S. Roorda, T. van Dillen, A. Polman, C. Graf, A. van Blaaderen, and B.J. Kooi, Aligned gold nanorods in silica made by ion irradiation of core-shell colloidal particles, Adv. Mater. 16, 235 (2004).

[4] E. Snoeks, A. van Blaaderen, T. van Dillen, C.M. van Kats, M.L. Brongersma, and A. Polman, Colloidal Ellipsoids with Continuously Variable Shape, Advanced Materials 12, 1511 (2000).

[5] S. Klaumunzer, Modification of nanostructures by high-energy ion beams, Nucl. Instrum. Methods Phys. Res. B 244, 1 (2006).

[6] C. Harkati Kerboua, J.M. Lamarre, M. Chicoine, L. Martinu, and S. Roorda, Elongation of gold nanoparticles by swift heavy ion irradiation: Surface plasmon resonance shift dependence on the electronic stopping power, Thin Solid Films 527, 186 (2013).

[7] A.A. Leino, O.H. Pakarinen, F. Djurabekova, K. Nordlund, P. Kluth, and M.C. Ridgway, Swift Heavy Ion Shape Transformation of Au Nanocrystals Mediated by Molten Material Flow and Recrystallization, Materials Research Letters 2, 37 (2014).

[8] H. Amekura, P. Kluth, P. Mota-Santiago, I. Sahlberg, V. Jantunen, A.A. Leino, H. Vazquez, K. Nordlund, F. Djurabekova, N. Okubo, and N. Ishikawa, Vaporlike phase of amorphous $\mathrm{SiO}_{2}$ is not a prerequisite for the core/shell ion tracks or ion shaping, Phys. Rev. Mater. 2, 096001 (2018).

[9] M.P. Allen and D.J. Tildesley, Computer Simulation of Liquids (Oxford University Press, Oxford, England, 1989).

[10] K. Nordlund, PARCAS computer code (2016). The main principles of the molecular dynamics algorithms are presented in $[10,11]$. The adaptive time step and electronic stopping algorithms are the same as in [12]. The code is published as part of [13].

[11] K. Nordlund, M. Ghaly, R.S. Averback, M. Caturla, T. Diaz de la Rubia, and J. Tarus, Defect production in collision cascades in elemental semiconductors and fcc metals, Phys. Rev. B 57, 7556 (1998).

[12] M. Ghaly, K. Nordlund, and R.S. Averback, Molecular dynamics investigations of surface damage produced by kiloelectronvolt self-bombardment of solids, Philosophical Magazine A 79, 795 (1999).

[13] K. Nordlund, Molecular dynamics simulation of ion ranges in the 1-100 keV energy range, Computational Materials Science 3, 448 (1995).

[14] F. Granberg, K. Nordlund, M.W. Ullah, K. Jin, C. Lu, H. Bei, L.M. Wang, F. Djurabekova, W.J. Weber, and Y. Zhang, Mechanism of Radiation Damage Reduction in Equiatomic Multicomponent Single Phase Alloys, Phys. Rev. Lett. 116, 135504 (2016). 
[15] K. Nordlund, M. Ghaly, and R.S. Averback, Mechanisms of ion beam mixing in metals and semiconductors, Journal of Applied Physics 83, 1238 (1998).

[16] A.A. Leino, O.H. Pakarinen, F. Djurabekova, and K. Nordlund, A study on the elongation of embedded Au nanoclusters in SiO2 by swift heavy ion irradiation using MD simulations, Nuclear Instruments and Methods in Physics Research Section B: Beam Interactions with Materials and Atoms 282, 76 (2012).

[17] H. Vázquez, E.H. Åhlgren, O. Ochedowski, A.A. Leino, R. Mirzayev, R. Kozubek, H. Lebius, M. Karlušic, M. Jakšic, A.V. Krasheninnikov, J. Kotakoski, M. Schleberger, K. Nordlund, and F. Djurabekova, Creating nanoporous graphene with swift heavy ions, Carbon 114, 511 (2017).

[18] M. Toulemonde, W. Assmann, C. Dufour, A. Meftah, F. Studer, and C. Trautmann, Experimental phenomena and thermal spike model description of ion tracks in amorphisabel inorganic insulators, Mat. Fys. Medd. Dan. Vid. Selsk. 52, 263 (2006).

[19] O.H. Pakarinen, F. Djurabekova, and K. Nordlund, Density evolution in formation of swift heavy ion tracks in insulators, Nuclear Instruments and Methods in Physics Research Section B: Beam Interactions with Materials and Atoms 268, 3163 (2010).

[20] F. Djurabekova and K. Nordlund, Atomistic simulation of the interface structure of Si nanocrystals embedded in amorphous silica, Phys. Rev. B 77, 115325 (2008).

[21] S. Munetoh, T. Motooka, K. Moriguchi, and A. Shintani, Interatomic potential for Si-O systems using Tersoff parameterization, Computational Materials Science 39, 334 (2007).

[22] P. Erhart, N. Juslin, O. Goy, K. Nordlund, R. Müller, and K. Albe, Analytic bond-order potential for atomistic simulations of zinc oxide, Journal of Physics: Condensed Matter 18, 6585 (2006).

[23] A. Slablab, T.J. Isotalo, J. Mäkitalo, L. Turquet, P.-E. Coulon, T. Niemi, C. Ulysse, M. Kociak, D. Mailly, G. Rizza, and M. Kauranen, Fabrication of Ion-Shaped Anisotropic Nanoparticles and their Orientational Imaging by Second-Harmonic Generation Microscopy, Scientific Reports 6, 37469 (2016).

[24] S. Klaumunzer, Ion tracks in quartz and vitreous silica, Nucl. Instrum. Methods Phys. Res. B 225, 136 (2004).

[25] T. van Dillen, E. Snoeks, W. Fukarek, C.M. van Kats, K.P. Velikov, A. van Blaaderen, and A. Polman, Anisotropic deformation of colloidal particles under MeV ion irradiation, Nuclear Instruments and Methods in Physics Research Section B: Beam Interactions with Materials and Atoms 175-177, 350 (2001).

[26] S. Klaumunzer, Ion hammering of silica colloids, Nucl. Instrum. Methods Phys. Res. B 215, 345 (2004).

[27] E.A. Dawi, A.M. Vredenberg, G. Rizza, and M. Toulemonde, Ion-induced elongation of gold nanoparticles in silica by irradiation with $\mathrm{Ag}$ and $\mathrm{Cu}$ swift heavy ions: track radius and energy loss threshold, Nanotechnology 22, 215607 (2011).

[28] H. Amekura, N. Ishikawa, N. Okubo, M.C. Ridgway, R. Giulian, K. Mitsuishi, Y. Nakayama, Ch. Buchal, S. Mantl, and N. Kishimoto, Zn nanoparticles irradiated with swift heavy ions at low fluences: Optically-detected shape elongation induced by nonoverlapping ion tracks, Phys. Rev. B 83, 205401 (2011).

[29] H. Amekura, N. Okubo, D. Tsuya, and N. Ishikawa, Counterevidence to the ion hammering scenario as a driving force for the shape elongation of embedded nanoparticles, AIP Advances 7, 085304 (2017).

[30] H. Amekura, S. Mohapatra, U.B. Singh, S.A. Khan, P.K. Kulriya, N. Ishikawa, N. Okubo, and D.K. Avasthi, Shape elongation of Zn nanoparticles in silica irradiated with swift heavy ions of different species and energies: scaling law and some insights on the elongation mechanism, Nanotechnology 25, 435301 (2014).

[31] A. Benyagoub, S. Loeffler, M. Rammensee, and S. Klaumunzer, Ion-beam-induced plastic deformation in vitreous silica, Radiation Effects and Defects in Solids 110, 217 (1989).

[32] H. Amekura et al., unpublished. 
354 [33] Y. Cherednikov, S.N. Sun, and H.M. Urbassek, Hybrid particle-in-cell/molecular dynamics simulation of swift-ion tracks in LiF, Phys. Rev. B 87, 245424 (2013).

[34] G. Rizza, P.E. Coulon, V. Khomenkov, C. Dufour, I. Monnet, M. Toulemonde, S. Perruchas, T. Gacoin, D. Mailly, X. Lafosse, C. Ulysse, and E.A. Dawi, Rational description of the ion-beam 358 359 shaping mechanism, Phys. Rev. B 86, 035450 (2012). 


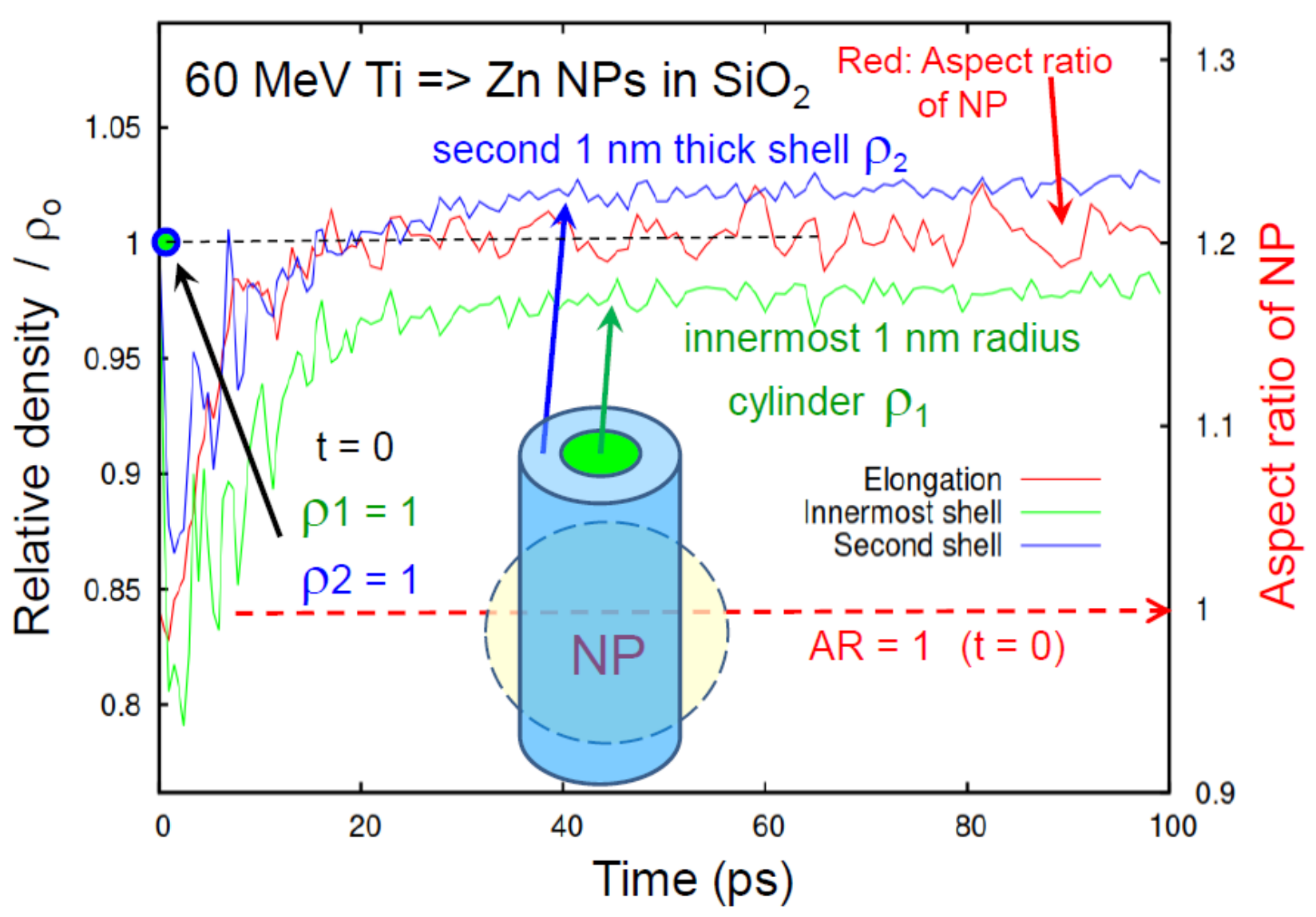

366 Fig. 1. Time evolutions of the relative density of amorphous $\mathrm{SiO}_{2}$ in the innermost cylinder of $1 \mathrm{~nm}$ 367 radius along the ion trajectory (blue) and in the second shell of $1 \mathrm{~nm}$ thick (green) after an impact of 60 $368 \mathrm{keV}$ Ti ion to silica including NP, which are calculated from MD simulations. A red curve shows that 369 of the calculated aspect ratio of NP in the right axis scale. It should be noted that the radius of NP is 6 $370 \mathrm{~nm}$, which is much larger than the hypothetical core/shell track (green and blue) of $2 \mathrm{~nm}$ in radius. 


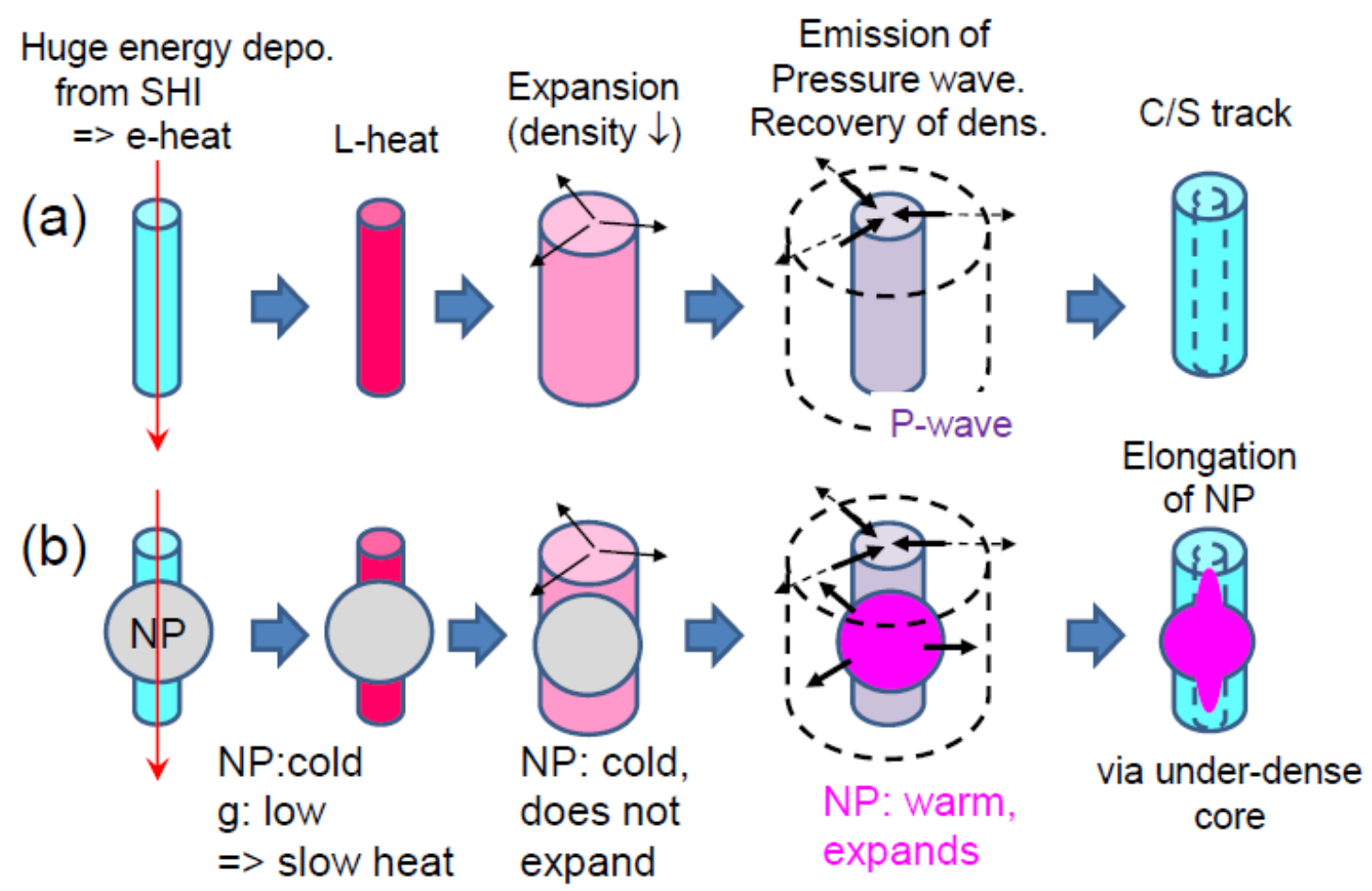

376 Fig. 2. The upper row (a) shows schematically depicted images of the temporal evolution of amorphous 377 silica after a SHI impact, i.e., the C/S track formation. The lower row (b) shows that of a NP in silica 378 after the SHI impact, i.e., the elongation of NP. The evolutions shown here are based on not only the 379 calculated results from the MD simulations but also physical reasoning. 